\title{
PERCEPCIONES SOBRE EL CLIMA DE LA COMUNICACIÓN (CCO): INSPECCIÓN A LAS PYMES TURÍSTICAS EN UN ENTORNO COMPETITIVO',2,3
}

Communication Climate (CC) Perceptions: Inspection of tourism SMEs in a competitive environment

Fecha de recepción: 4 de mayo de 2020 Fecha de aceptación: 10 de junio de 2020

1- José Guadalupe Magdaleno Elizondo. Grado académico: Maestría en Administración Campo Finanzas. Adscripción: Universidad Autónoma de Aguascalientes. Correo electrónico: jose.magdaleno@edu.uaa.mx. ORCID ID: https://orcid. org/0000-0002-5807-7815

2- Ismael Manuel Rodríguez Herrera. Grado académico: Doctorado en Turismo. Adscripción: Universidad Autónoma de Aguascalientes. Correo electrónico: imrodri@correo.uaa.mx. ORCID ID: https://orcid.org/ 0000-0001-5007-1323

3- Diana Elizabeth Hernández-Ochoa. Grado académico: Maestría en Administración. Adscripción: Universidad Autónoma de Aguascalientes. Correo electrónico: dianahernande37@hotmail.com. ORCID ID: https://orcid.org/0000-0002-6213$283 \mathrm{X}$

nR José Guadalupe Magdaleno Elizondo, Ismael Manuel Rodríguez Herrera y Diana Elizabeth Hernández-Ochoa 


\title{
RESUMEN
}

En la actualidad, el creciente interés por el estudio de las pequeñas y medianas empresas (Pymes) del sector turístico ha propiciado un aumento en el desarrollo de investigaciones que abordan todo tipo de relaciones y variables. Sin embargo, el clima de la comunicación (CCO) es un elemento que se ha estudiado poco en ese contexto. El presente trabajo pretende atender esa brecha, por medio de un cuestionario aplicado a 260 empresas de dicho sector. Con la finalidad de explorar la relación entre la confianza (COF), la participación en la toma de decisiones (PTD), el apoyo (AP), la apertura en la comunicación descendente (ACD) y ascendente (ACA) y el interés por las metas de alto impacto (IMA) con respecto del (CCO). Los resultados indican que todas las variables inciden de forma positiva-significativa en el (CCO). Algunas implicaciones se discuten.

Palabras clave: Clima de Comunicación, Información, Industria del Turismo, Pymes

\begin{abstract}
Currently, the growing interest in the study of small and medium-sized enterprises (SME's) in the tourism sector has led to an increase in the development of research that addresses all kinds of relationships and variables. However, the communication climate (CC) is an element that has been scarcely studied in this context. This paper aims to address this gap, through a questionnaire applied to 260 companies in the sector. With the intention of exploring the relationship between trust (TT), participation in decision-making (PDM), support (SUP), openness in downward communication (ODC) and upward communication (OUC) and interest in high impact goals (IHG) with respect to (CC). The results indicate that all the variables include the positive-significant form in the (CC). Some implications are discussed.
\end{abstract}

Keywords: Communication Climate, Information, Tourism Industry, SMEs

Clasificación JEL: D83, L83, Z31

Introducción 

1 estudio de la comunicación dentro del ámbito organizacional es un elemento que lleva ya varias décadas presente en distintos contextos y cuyo papel ha ido cobrando cada vez más importancia dentro del desarrollo y planeación de estrategias que encaminan a las organizaciones a obtener mejores resultados en términos de desempeño (Snyder y Morris, 1984). Esto se da porque la esencia de la comunicación radica en la recopilación de información sobre un contexto determinado (Trombetta y Rogers, 1988). Elemento que Guzley (1992) consideró independiente del clima organizacional, ya que es un elemento aislado que solo involucra fenómenos relacionados con la comunicación. Dicho autor determinó que el clima de la comunicación era un elemento que comúnmente se asociaba con variables individuales (compromiso, motivación, satisfacción laboral, etc.) y que su relación con diversos factores organizacionales generalmente era moderada por otras variables.

Es cierto que algunas variables organizacionales e individuales influyen en la percepción sobre el clima dentro de una empresa, al igual que ciertos elementos internos y externos, mismos que pueden afectar el comportamiento de los miembros de trabajo puesto que, a raíz del clima, los empleados pueden determinar lo que se espera de ellos, así como las actitudes y comportamientos que estos deben de tener dentro de su ambiente de trabajo (Brunet, 1992). La importancia por el uso de diversas variables relacionadas a la comunicación enmarca un sinfín de posibilidades en torno a las distintas actividades dentro de la operación de una empresa. Tal como se desarrolla en la investigación de Álvarez (2007), donde se prueba que estos elementos han probado ser un excelente aliado para alcanzar el éxito organizacional, incluso dicho autor encontraba que el aprovechamiento de diversos factores dentro de la comunicación se puede traducir en beneficios económicos y en ventajas dentro del proceso de gestión dentro de la empresa.

En la actualidad, las organizaciones se encuentran ante un mar de retos propios a cada uno de los mercados a los que van dirigidos, ante esto, las empresas han ido evolucionando hacia un nuevo paradigma organizacional que involucra una preocupación verdadera por el desarrollo de una organización con mayor grado de sensibilidad, tratando de armonizar entre los intereses sociales y empresariales (Arráez, Osuna y Peris, 2014). Esto ha permitido que las organizaciones tomen en cuenta elementos que anteriormente eran pasados por alto y que poco a poco elementos de la comunicación, de la cultura y del contexto mismo cobren especial importancia para encaminar a las empresas a entornos más competitivos (Barney, 1991 y 1986; Hernández, Mendoza y González, 2008; Krajnakova, Navikaite y Navickas, 2015).

Es por esto que es posible evidenciar que el estudio de dichos elementos de la comunicación en el ambiente de las empresas turísticas es relativamente nuevo (comparando otro tipo de variables organizacionales) y sin embargo, dichos elementos han ido cobrando cada vez mayor importancia puesto que una parte esencial de la comunicación subyace en lo más profundo de la organización (en compañía de los valores, la misión, la cultura, etc.) (Kahle, 2002).

Por todo lo anterior, es importante considerar los múltiples beneficios que obtendrían las pequeñas y medianas empresas (Pymes) por el adecuado uso de diversas variables relacionadas con el clima de la comunicación, mismas que han permitido que estas organizaciones puedan explotar su verdadero potencial competitivo en términos del aumento en su desempeño (Mbatha, 2012). Otro beneficio se centra en el aprovechamiento de la unificación de significados, ya que estos elementos permiten que las empresas obtengan un mejor ambiente de colaboración, proactividad y de actitudes que fomentan el correcto desarrollo de la empresa (Zach, 2013). Si bien el personal directivo/gerencial es el encargado de establecer 
el tipo de clima de la comunicación dentro de la empresa, se puede indicar que aquellas empresas con un ambiente de apoyo, que fomente la participación y la flexibilidad en la resolución de conflictos obtendrá mejores beneficios en términos del desarrollo y desempeño tanto a nivel individual-grupal como organizacional, que aquellas organizaciones cuya orientación a dicho clima sea más conservadora (Nordin, Sivapalan, Bhattacharyya, Ahmad y Abdullah, 2014).

Para poder comprender dichos elementos, en la presente investigación se lleva a cabo la aplicación del modelo derivado de la línea de investigación propuesta por Peterson y Pace (1976), en la que se aborda al clima de la comunicación (CCO), mismo que se presume se encuentra asociado con las variables (1) Confianza (COF), (2) Participación en la toma de decisiones (PTD), (3) Apoyo (AP), (4) Apertura en la comunicación descendente (ACD), (5) Apertura en la comunicación ascendente (ACA) y finalmente el (6) Interés en las metas de alto impacto (IMA); dicha línea de investigación plantea que dichas variables independientes son necesarias para estimular al clima de la comunicación (CCO).

Para llevar esto acabo se realiza un análisis de regresión a cada uno de los factores (COF, PTD, AP, ACD, ACA, IMA) con respecto del constructo (CCO), a pesar de existir un amplio cuerpo de literatura que aborda la relación entre diversos elementos de la comunicación y del (CCO) existe una brecha sobre la correspondencia teórica-empírica entre dichas variables, así como la relación de estas en el contexto de las Pymes del sector turístico dentro del estado de Aguascalientes.

Para tratar de aportar explicación a dicha problemática, primeramente, se desarrolla un análisis de la literatura para cada una de las variables establecidas, posterior a ello se realiza una breve explicación del modelo y de los competentes que lo integran, seguido del desarrollo de la propuesta de descripción y diseño del modelo de investigación, finalmente, en las últimas secciones se encuentra un análisis y discusión sobre los resultados, la discusión y las conclusiones sobre estos.

\section{Revisión de literatura}

\subsection{El clima organizacional}

Dadas las condiciones económico-sociales de los últimos años, mismas que han propiciado que las organizaciones busquen estrategias que les permitan desarrollarse de una forma más competitiva (con respecto de su competencia), han fomentado el estudio del comportamiento de los individuos en las organizaciones (Aburto y Bonales, 2011). Todas las organizaciones tienen características o propiedades que las hacen únicas (Brunet, 2007), entre esas características se cuenta el ambiente interno formado por las personas que integran la organización, lo cual es considerado como parte del clima organizacional (Robbins, 1999; Sandoval-Caraveo, 2004; Silva, 1996).

Las primeras publicaciones que se realizaron sobre el clima organizacional se remontan a la segunda mitad del siglo XX (Brunet, 1992), donde un primer enfoque apuntaba a que el clima es producto de la unión de diversos factores organizacionales que interactúan con los miembros de la empresa y de los cuales, se forman vínculos que a su vez se encuentran determinados por diversas características externas que se viven tal cual son percibidas por el trabajador (Guillén del Campo, 2013). Propiamente se consideraba que el clima es el producto de la percepción que tiene un miembro de su empresa, con respecto

nR José Guadalupe Magdaleno Elizondo, Ismael Manuel Rodríguez Herrera y Diana Elizabeth Hernández-Ochoa 
a todo lo que la integra (Halpin y Croft, 1963) o por aquellas características específicas que describe a una organización (propio a cada organización) (Forehand y Von Haller, 1964) o como aquello que es subjetivo dentro del sistema formal de la estructura (Litwin y Stringer, 1968) y que este gira alrededor de la estructura, políticas y reglas, hasta atributos subjetivos como la cordialidad y el apoyo (Dessler, 1993) y se manifiesta a través de los miembros de la organización por medio de manifestaciones culturales (Cabrera, 2003).

Tratar de definir este concepto de clima organizacional involucra el trabajar con un grupo de componentes y determinantes que ofrecen una visión global de la organización (Salazar, Guerrero, Machado y Cañedo, 2009). Es precisamente por este enfoque que se concibe al clima organizacional como el resultado de la interacción entre las características inherentes a las personas y de las propias de las organizaciones (Escuela del Partido «Nico López», s.f.). Diversos elementos han ido sufriendo modificaciones a lo largo del histórico del constructo en sí, puesto que estos se encuentran sujetos al contexto que se estudie (Duran, 2002; Escuela del Partido «Ñico López», s/f; Patterson, West, Shackleton, Dawson, Lawthom, Maitlis, Robinson y Wallace, 2005). Sin embargo, se coincide en que de forma común estos son: El ambiente físico, las características estructurales, el ambiente social, las características personales y el comportamiento organizacional.

El propósito de la evaluación del clima organizacional es el logro de un acercamiento a la realidad cotidiana de la empresa, la identificación de las áreas donde se estén generando conflictos, ya que esto pudiera reducir la eficiencia en el desenvolvimiento usual de centro de trabajo y hacer frente a costos inesperados en un futuro (Guillén del Campo, 2013). Propiamente, algunas de las dimensiones básicas que se han identificado son; la motivación, liderazgo, reciprocidad y participación, las cuales, a su vez, se subdividen en categorías o subvariables (Cabrera, 2003; Cruz, 2007; Rodríguez, Flores y García, 2006).

De forma general, a lo largo de su historia se ha evidenciado que para tener un clima organizacional adecuado una organización debe de poseer elementos como: recompensas, riegos y toma de decisiones, apoyo y tolerancia (Litwin y Stringer, 1968), así como de apertura, comunicación fluida y motivación (Bowers y Taylor, 1970), igualmente debe incluir autonomía, cooperación y flexibilidad (Pritchard y Karasick, 1973), al igual que métodos de mando e influencia en la planificación y control organizacional (Likert y Likert, 1986), finalmente debe de tener grado de estructura, consideración y agradecimiento (Brunet, 2011).

\subsection{La comunicación organizacional}

Sin lugar a dudas, la comunicación es un elemento vital para las organizaciones, por ello, este elemento ha estado presente dentro de la comunidad científica desde hace varias décadas (Krohn, 1981). Distintos autores se han dado a la tarea por tratar de comprender las características que hacen que tener una comunicación efectiva pueda derivar en alguna mejora (Patterson, 2016) y que esta mejora pueda impactar en el desarrollo de una empresa dedicada al servicio (Ottenbacher y Gnoth, 2005). Antes de entrar en términos del proceso de comunicación es conocer la diferencia sustancial que existe entre información y comunicación, la primera es descrita como datos que son almacenados, mientras que la última es definida como un proceso mediante el cual dicha información es transferida 
y comprendida por el receptor (Rodríguez, 2003).

Es bien sabido que la comunicación se compone de ocho actores (Robbins y Judge, 2013) que poseen funciones específicas mediante las cuales se logra llevar a cabo el proceso de comunicación, entre ellos se encuentra: El emisor, quien se encarga de lanzar una señal o mensaje, mismo que es enviado bajo una codificación por medio de un canal (formal o informal), para así llegar a un receptor, quien es capaz de recibirlo. Es tarea del receptor decodificar el mensaje, es decir, de comprenderlo y darle sentido. Dichos autores discuten sobre la observación de la existencia de barreras que pudieran dificultar el envío y recepción de los mensajes, a estas barreras las denominan 'ruido' y pueden ser diferencias culturales, dificultades semánticas, entre otras. La forma en que es posible tener certeza de que el mensaje ha alcanzado y ha sido entendido es cuando existe retroalimentación entre el emisor y el receptor.

Parte esencial del proceso de comunicación se encuentran al principio y al termino de dicho proceso, ya que primeramente es necesario tener ideas o información que se desean compartir (Robbins y Coulter, 2002) y otro elemento esencial subyace en la importancia que tiene el hecho de que el mensaje que es transmitido sea comprendido (Phillips y Brown, 1993). Durante el proceso de comunicación organizacional clásico, la comunicación prevalecía hacia una sola dirección, es decir, no era posible refutar o dar respuesta a los altos mandos, quienes solo entregaban notificaciones a los puestos inferiores sin ofrecer oportunidades de diálogo (Moreno, Arbeláez y Calderón, 2014).

Los orígenes de la comunicación organizacional se remontan a la década de 1970, cuando comenzó a verse desde un punto de vista teórico, principalmente en Europa y Estados Unidos (Segovia, 1999). Se ha considerado la comunicación institucional (presente en instituciones públicas) u organizacional (propia de las empresas), se manifiesta cuando es necesario mantener actividades de manera que, tanto en los públicos internos como externos, la comunicación sea estable (Arráez et al., 2014). Es por esto que Fernández (1997) abordaba a la comunicación organizacional como el empleo de técnicas y actividades que estimulan el flujo de mensajes entre los miembros de la organización y entre ésta y su medio, además de influir en opiniones, aptitudes y conductas de los públicos, para así cumplir con los objetivos trazados por las organizaciones.

En la actualidad, la comunicación organizacional ha dejado de ser vista únicamente como el envío de mensajes hacia el exterior o que los mensajes se muevan de forma descendente a nivel interno, puesto que esta alcanzó un punto en el que fue necesaria una participación más humana (desde la perspectiva de la interacción social), por lo que fue necesario estimularla en forma de trabajo colaborativa sin entorpecer la creatividad (Rebeil, 2006). El trabajo de la comunicación organizacional debe ir encaminado a apoyar la productividad de la organización, por lo que es necesario comprender como es que este concepto (abstracto) de comunicación puede aportar a diversos elementos (concretos) organizacionales, ante ello Nosnik (1998 y 2003) apunta a que dicho proceso de comunicación debe tener elementos productivos, dinámicos y lineales. Finalmente, el autor concluye que con el adecuado uso de estos elementos dentro del proceso de comunicación organizacional es posible lograr una mayor productividad (Soria, 2008). Sin embargo, diversos autores discuten sobre la posibilidad de que existen varios tipos de comunicación organizacional, (e.g.) Nosnik (1996) señala que esta puede ser abierta, evolutiva, flexible e instrumentada. Mientras que Pérez (2000) indica que esta puede darse por medio de la comunicación operativa, táctica y estratégica.

Particularmente la comunicación dentro del nivel operativo puede darse de dos maneras (formal o 
informal) y cada una de estas tiene sus propias características, esto puede afectar significativamente la manera en que la que el mensaje puede ser comprendido (Parra y Rincón, 2009) (e.g.):

- La comunicación formal: Sigue la estructura de la organización. Es la forma en que los niveles superiores o administradores se transmiten la información al personal de la organización (Gómez y Sarsosa, 2011).

- La comunicación informal: los mensajes pueden ser transmitidos fuera de la estructura de la organización (Daradjat y Sugiyanto, 2017; Parra y Rincón, 2009), además, resulta de la convivencia natural entre toda la planta laboral (Sanchis y Bonavia, 2017) y ayuda a que los mensajes compartidos sean menos complejos (Kwofie, Aigbavboa y Machethe, 2019).

Si se considera que el proceso de comunicación se desarrolla de forma interna en la organización misma que puede darse de forma interna (Ivancevich, Konopaske y Matteson, 2006) y externa (Bartoli, 1992; Cabrera, 2015). La primera debe de mantener una convivencia armoniosa pretendiendo lograr identidad y cultura corporativa (Cervera, 2004), de igual forma esta puede darse en diversos sentidos apoyada por herramientas análogas y digitales (Álvarez, 2007), esta se hace por medio de mensajes que son enviados de manera recíproca entre la estructura (Montoya, 2009), mediante los cuales se facilita la transferencia de información entre diversos miembros (Mazzei, 2010; Moreno et al., 2014) y tiene la finalidad de contribuir a la colaboración, información, integración y motivación del personal entero (Peña-Acuña, Caldevilla-Domínguez y Batalla, 2017).

Mientras que la segunda refiere al proceso de comunicación que se desarrolla más allá de los límites de la organización (Cabrera, 2015) y se caracteriza por ser externa operativa, estratégica, por ofrecer notoriedad, por ser no intencional y de obligada aportación (Bartoli, 1992). Por todo lo anterior, es fundamental considerar la importancia que tiene la comunicación interna dentro de la organización, puesto que esta es la encargada de transmitir todos los mensajes a lo largo de la estructura de la empresa (indistintamente de la orientación por la que fluye la información), por ello, es esencial tomarla en cuenta como un elemento trascendente para el correcto desempeño de las empresas.

\subsection{El clima de comunicación organizacional}

$\mathrm{Al}$ igual que con diversos componentes organizacionales intangibles dentro de la gestión empresarial, el clima de la comunicación es un elemento que ha sufrido diversos cambios en la conceptualización de este a través de su histórico, lo anterior se ha dado puesto que las diversas concepciones que se tienen sobre este han permitido adaptarlo a contextos que previamente no se habían abordado. En el caso de la línea de investigación desarrollada por Pace y Faules (1993 y 2010) quienes indicaban que este clima se produce como resultado de la combinación de las percepciones sobre los eventos de comunicación, al igual que por medio de los comportamientos, de las interacciones, de las relaciones interpersonales y de las oportunidades de crecimiento en la organización.

nR José Guadalupe Magdaleno Elizondo, Ismael Manuel Rodríguez Herrera y Diana Elizabeth Hernández-Ochoa 
Este clima de comunicación organizacional es fundamental cuando: la organización busca que los empleados sientan apoyo, usen la libertad de expresión, consideren mayor proximidad entre departamento y se mantengan motivados a trabajar en equipo para alcanzar los objetivos planteados (Hassan y Maqsood, 2012; Peterson y Pace, 1976). Inclusive, se ha evidenciado los posibles beneficios sobre el desempeño y su relación con distintos elementos dentro de la comunicación organizacional que traen las retroalimentaciones, así como el desarrollo de un clima de comunicación que apoye a los miembros de la organización (Brownell, 1994). Mientras que otra corriente de investigación indica que el clima de la comunicación involucra únicamente aspectos referidos al proceso de comunicación y sus elementos dentro de lo que ocurre en la organización (Smidts, Pruyn y Van Riel, 2001), considerando como la percepción de los distintos miembros de la estructura, la apertura y la facilidad de interacción que estos tienen entre sí, al igual que la confianza sobre la información diseminada. En esa misma obra, los autores añaden que es preciso distinguir el alcance del clima de la comunicación, apoyándose en el trabajo de Jones y James, recalcaban la importancia de separar lo que es el clima psicológico del organizacional, puesto que "el primero refiere a lo que el individuo percibe, involucrando sus percepciones e interpretaciones de su ambiente laboral, a nivel psicológico (sentido y significado) (...) mientras que el segundo refiere al clima psicológico compartido" (p. 1053).

Estudios previos han mostrado la importancia del vínculo entre el (CCO) y diversas variables individuales y organizacionales (e.g.) la motivación de los empleados hacia el logro de objetivos de alto rendimiento y el que participen durante la toma de decisiones (Noknoi, Boripunt y Ngowsiri, 2011; Sager, 2015; Weihrich y Koontz, 1993), así como la literatura permite observar, también, la relación entre el (CCO) y el apoyo percibido por el empleado, además de la confianza y apertura de expresión de ideas y sentimientos (Hassan y Maqsood, 2012; Neves y Eisenberger, 2012; Park y Kim, 2012; Petersen, 2005; Uysal, 2019). Para que esta relación se pueda dar de una forma más fluida, es necesario que las empresas del sector turístico aprovechen todos los medios y las formas de comunicación posibles entre la estructura organizacional, una manera eficiente para realizar esto, es por medio de la comunicación interna, donde es necesario que la estructura pueda compartir información confiable y precisa sobre la operación de la organización, bien sea por medio formales o informales (Chan, Chow, Loi y Xu, 2017).

Como se ha puesto en evidencia, el (CCO) es el ambiente existente dentro de la organización, así como la manera en la que se dan las relaciones laborales, este abarcando las formas de comunicación (verbalno verbal), con respecto de la manera en la que se relaciona la estructura organizacional (independiente de la orientación que tenga el flujo de información). Por ello es importante considerar aquellos elementos que integran a dicho concepto, puesto que esto facilitaría enormemente la identificación sobre cuáles son los aspectos con mayor relación a dicho constructo (CCO).

\subsubsection{Confianza (COF)}

La confianza es uno de los elementos principales dentro del clima de comunicación, porque si se considera que el (CCO) es todo aquello que se puede relacionar con el ambiente y los fenómenos de comunicación dentro de la organización, la (COF) es un elemento fundamental, puesto que esta refleja las preocupaciones verdaderas de los miembros de la estructura por sus pares y la organización (Pincus, 
Knipp y Rayfield, 1990). Es precisamente esta confianza la que determina las expectativas que tienen los miembros de la organización sobre sus relaciones interpersonales y de trabajo y es la responsable de que de forma individual o grupal existan comportamientos predeterminados ante ciertas situaciones o fenómenos y sin ella es complicado que los individuos o los grupos puedan alcanzar de una forma sencilla las metas y los objetivos organizacionales (Diallo y Thuillier, 2005).

La relación entre la comunicación y la confianza ha sido muy estrecha desde hace muchos años, puesto que la primera es alusiva a la manera en la que la información es transmitida entre los miembros de la empresa, mientras que la segunda integra la expectativa sobre que la información que se comparte es confiable (Zeffane, Tipu y Ryan, 2011). Por ello, es importante considerar la unificación de esfuerzos y creencias dentro de la estructura organizacional, puesto que es importante fortalecer las relaciones interpersonales, ya que se pretende generar ambientes íntegros, creíbles y seguros. Debido a eso, se desglosa la primera hipótesis de investigación, misma que plantea:

H1: Existe relación positiva significativa entre la confianza (COF) y el clima de la comunicación (CCO) en las empresas del sector turístico dentro del estado de Aguascalientes.

\subsubsection{Participación en la toma de decisiones (PTD)}

Otro de los principales componentes del clima de la comunicación es la participación en la toma de decisiones, elemento que es importante debido a que en cierto sentido el (CCO) es el ambiente en el que se pueden desarrollar suposiciones y actitudes, mismas que pueden determinar la manera en la que actúan tanto el personal directivo/gerencial como operativo, de la misma manera estos elementos repercuten en: la participación activa de los miembros de la organización, la satisfacción laboral y el desempeño del individuo o del grupo, para evitar malos resultados es preciso considerar el flujo de la información, la formación de metas/objetivos y elementos de control (Falcione, 1974). Por su parte, Wheeless, Wheeless y Howard (1983) identificaban que este factor se consideraba como la capacidad que tienen los miembros de la organización para poder participar de forma activa dentro del proceso de la toma de decisiones, en dicha investigación los autores observan que elementos como el conflicto, la proactividad en los procesos, la satisfacción laboral y elementos inherentes al individuo como la motivación eran los principales componentes beneficiados de la inclusión del personal al proceso de toma de decisiones.

Mientras que Stewart, Gudykunst, Ting-Toomey y Nishida (1986) encontraban que el estilo del personal directivo/gerencial impactaba en la apertura y la satisfacción laboral, esto supone la posibilidad de fomentar ciertos comportamientos que encaminan tanto al individuo como al grupo a realizar actividades que beneficien particularmente a dichos elementos (por medio de climas flexibles y sistemas abiertos). Por ello, es importante considerar que por medio de dicho proceso se pueden obtener múltiples beneficios, para lo cual, el personal directivo/gerencial deberá de encaminar sus estrategias de gestión para contemplar a la gran mayoría de los miembros de la organización en la toma de decisiones. Con ello en mente, se desglosa la primera hipótesis de investigación, misma que plantea: 
H2: Existe relación positiva significativa entre la participación en la toma de decisiones (PTD) y el clima de la comunicación (CCO) en las empresas del sector turístico dentro del estado de Aguascalientes.

\subsubsection{Apoyo (AP)}

Otro elemento importante para el desarrollo de un buen clima de la comunicación es el apoyo, puesto que este elemento permite moldear ciertos comportamientos, estudios previos encontraban que dependiendo del enfoque del (CCO) que tuviera la organización (considerando un enfoque de apertura y otro más conservador) en términos de flexibilidad sobre el apoyo entre la estructura, particularmente era posible identificar que aquellos climas con mayor apertura aumentan la interacción y el interés por alcanzar metas y objetivos (Rosenfeld, 1983). Es importante que las organizaciones tiendan a desarrollar ambientes con un mayor grado de apoyo entre los miembros de la estructura, por ello, deberán de considerar fomentar comportamientos que generen provisionalísimo, empatía, igualdad, espontaneidad, entre otros (Keenan, 1988).

Además, aquellas organizaciones que fomenten dicha variable tendrán mejores resultados en términos de conexiones interpersonales fuertes, buenos flujos de información, un adecuado manejo de la información (independientemente de la orientación), reduce el conflicto y promueve la cooperación y la cohesión dentro de la empresa (Wei y Morgan, 2004). Finalmente, las organizaciones deben de tomar en cuenta que el apoyo es un elemento vital para el correcto desarrollo del ambiente organizacional, puesto que esto ofrece múltiples beneficios para las organizaciones. Ante esto, se desglosa la siguiente hipótesis de investigación:

H3: Existe relación positiva significativa entre el apoyo (AP) y el clima de la comunicación (CCO) en las empresas del sector turístico dentro del estado de Aguascalientes.

\subsubsection{Apertura en la comunicación descendente (ACD) y Apertura en la comunicación ascendente (ACA)}

La apertura en la comunicación descendente es uno de los principales elementos dentro de la comunicación interna, que a su vez, conforman parte esencial dentro de clima de la comunicación, puesto que esta es la manera en la que el personal de jerarquía (lideres) interactúan con sus subordinados, este elemento cobra un rol vital dentro del (CCO) ya que, este tipo de comunicación puede determinar el comportamiento y la participación de los miembros de la organización (Nurlita, 2012). Esta misma autora encuentra a su vez que la comunicación ascendente es igual de importante que la descendente, puesto que estas se encuentran presentes dentro del proceso de la comunicación interna, elementos dentro del ambiente de la comunicación organizacional son vitales para el adecuado intercambio de información, propiamente si la comunicación ascendente es deficiente será difícil que el personal de jerarquía cuente con los elementos necesarios para un adecuado proceso de toma de decisiones. 
La apertura en la comunicación descendente es un elemento vital dentro del (CCO), puesto que si se considera que este último tiene un rol primordial en la motivación de los empleados, es menester que el personal directivo/gerencial ponga especial atención en el desarrollo de comportamientos que fomenten la apertura y que minimicen la filtración de información para con sus subordinados, ya que de esto depende que el ambiente de comunicación dentro de la empresa se desarrolle de una manera fluida y oportuna (Ramadanty y Martinus, 2016). Estos mismos autores indican que cuando la comunicación se invierte (en sentido ascendente) es entonces cuando elementos como la confianza, el apoyo, al igual que diversos comportamientos toman la iniciativa y cobran especial importancia con respecto del (CCO), si un empleado percibe que no existe apertura dentro del (CCO) entonces vera limitada su posibilidad de comunicar sus preocupaciones e intereses.

Es por ello por lo que es importante considerar que la apertura de la información por parte del personal de jerarquía hacia con sus subordinados es un elemento vital para el desarrollo de un ambiente adecuado para el intercambio de información, porque es importante que los miembros de la organización reciban de forma íntegra el mensaje y con ello las posibilidades de que el receptor pueda comprender de una mejor manera el mensaje aumentan, esto podría derivar en una mejora del rendimiento de la organización (Hidayat y Hanisha, 2019). Adicionalmente estos autores indican que la comunicación ascendente es un elemento fundamental para el desempeño del individuo y de la organización, señalan que en este tipo de comunicación elementos como: la confianza que se les tiene a los empleados (por parte de la jerarquía organizacional), la honestidad de los empleados, la apertura de los canales de comunicación, la capacidad y el interés por las necesidades de los miembros de la empresa, así como su motivación hacia los objetivos y las metas organizacionales de alto impacto son elementos que se relacionan entre este tipo de comunicación y el desempeño organizacional.

Por ello, es preciso que las organizaciones sean capaces de desarrollar esa flexibilidad y apertura por parte del personal de jerarquía hacia con los demás empleados, al igual que la manera en la que estos comparten información con sus superiores, tomando en cuenta la importancia porque ambos reciban de forma íntegra y oportuna la información que necesitan para el desarrollo de sus operaciones, ante esto se desglosan las siguientes hipótesis de investigación:

H4: Existe relación positiva significativa entre la apertura en la comunicación descendente (ACD) y el clima de la comunicación (CCO) en las empresas del sector turístico dentro del estado de Aguascalientes.

H5: Existe relación positiva significativa entre la apertura en la comunicación ascendente (ACA) y el clima de la comunicación (CCO) en las empresas del sector turístico dentro del estado de Aguascalientes.

\subsubsection{Interés en las metas de alto impacto (IMA)}

Finalmente, el interés en las metas de alto impacto es el último elemento que integra al (CCO), lo anterior permite identificar aquella disposición que tienen los miembros de la organización por alcanzar 
metas y objetivos determinados por la empresa, incluso se evidencia que con un clima de comunicación abierto es posible que los miembros de la estructura tengan mayor interés por alcanzar dichos objetivos, minimizando el conflicto (puesto que ponen los intereses de la organización por encima de los propios) (Hassan y Maqsood, 2012). Es por ello que todos los miembros de la organización (indistintamente de la jerarquía) deben de concentrar sus esfuerzos por lograr las metas y los objetivos de la organización, de la misma manera este factor requiere que la organización posea un (CCO) con un nivel alto de confianza, apertura y apoyo (especialmente en las relaciones interpersonales) (Mohammed y Hussein, 2013), con ello en mene se desglosa la siguiente hipótesis de investigación:

H6: Existe relación positiva significativa entre el Interés en las metas de alto impacto (IMA) y el clima de la comunicación (CCO) en las empresas del sector turístico dentro del estado de Aguascalientes.

\section{Metodología}

El presente trabajo se deriva de un proyecto de investigación de mayor tamaño, mismo que corresponde al desarrollo de una tesis doctoral, la cual, aborda diversos aspectos organizacionales y su interacción en el sector de las Pymes turísticas, en lo particular, el presente trabajo aborda únicamente el análisis sobre la situación que presentan esas empresas con respecto del clima de la comunicación en dicho sector económico. El modelo utilizado en este trabajo parte de la corriente de investigación originalmente propuesta por Peterson y Pace (1976), misma que aborda al clima de la comunicación como un elemento que puede ser medido por seis factores y doce items en total (cada factor está integrado por dos items): (1) Confianza (COF), (2) Participación en la toma de decisiones (PTD), (3) Apoyo (AP), (4) Apertura en la comunicación descendente (ACD), (5) Apertura en la comunicación ascendente (ACA), por último el (6) Interés en las metas de alto impacto (IMA).

Dicho cuestionario se aplicó a una muestra de 260 Pymes del sector turístico dentro del estado de Aguascalientes, cuya actividad económica tuviera relación con el turismo, al ocio o a la recreación. Dicho instrumento consta de seis factores, mismos que se integran por dos items cada uno, por medio de dicho instrumento es posible conocer la percepción del personal directivo/gerencial la relación que tienen dichas empresas con respecto del clima de la comunicación. Este cuestionario permitió obtener los elementos necesarios para el desarrollo de una base de datos, misma que cumple los parámetros para el análisis estadístico comprensivo para los subsectores turísticos contenidos en la muestra (hotelería, alimentos y bebidas, museos, bares, entre otros). Estos datos satisfacen los lineamientos de una regresión lineal, misma que pretende explicar la relación que guarda cada uno de los factores (COF, PTD, AP, ACD, ACA, IMA) con respecto del clima de la comunicación (CCO).

Gracias al análisis de la literatura, fue posible identificar aquellos elementos que integran al concepto del clima de la comunicación desde la perspectiva del desempeño, desarrollo, efectividad y productividad laboral. Esto permitió identificar cuáles de las variables determinadas presentaban mayor incidencia en dicho constructo, observando que el sector objetivo propiamente tiende a tener una mayor inclinación hacia la apertura en la comunicación descendente, así como un menor interés por el interés en las

nR José Guadalupe Magdaleno Elizondo, Ismael Manuel Rodríguez Herrera y Diana Elizabeth Hernández-Ochoa 
metas de alto impacto. Es del interés mencionar que la presente investigación se destaca, puesto que una buena cantidad de literatura se observan análisis de relaciones cuyo enfoque se centra particularmente en alguno de los elementos mencionados y su interacción con algún otro tipo de variable organizacional.

Esta escasez de análisis plantea una problemática sobre el desarrollo de apoyos empíricos que puedan soportar las relaciones encontradas particularmente con cualquiera de las variables presentes en este trabajo. Es por ello, que este trabajo se concentra en aportar evidencia que atienda esa brecha, mediante el planteamiento de diversas hipótesis de investigación, con el propósito de determinar y analizar las relaciones entre las variables y el clima de la comunicación.

\subsection{Modelos de regresión}

Para tratar de responder las hipótesis de investigación se hace uso de un análisis de regresión líneal, mismo que permite determinar y analizar la correspondencia y el sentido que esta tiene en la relación de cada una de las variables independientes (COF, PTD, AP, ACD, ACA, IMA) y el poder que ejercen en el (CCO) en el contexto de las Pymes del sector turístico dentro del estado de Aguascalientes. Este trabajo se presume de tipo no experimental transeccional (puesto que no se ejerce control sobre ninguna de las variables), el estudio se realiza a un grupo de 260 Pymes del sector turístico de las que se incluyen hoteles, agencias de viajes, negocios de alimentos y bebidas, spa (s) y aquellas empresas cuya actividad económica se relacione con el sector turístico dentro del estado de Aguascalientes.

El cuestionario utilizado proviene de la línea de investigación desarrollada por Peterson y Pace (1976), misma que plantea que el (CCO) puede ser medido por seis dimensiones, dicho cuestionario fue aplicado al personal directivo/gerencial e incluso a los dueños de las empresas (según fuera el caso), con el objetivo de indagar sobre su percepción de las variables determinadas en su lugar de trabajo, dicha encuesta cuenta con un total de doce items y un rango de respuestas en escala Likert a cinco puntos (desde $1=$ Totalmente en desacuerdo, hasta $5=$ Totalmente de acuerdo).

Las variables utilizadas (v) para estimar los modelos regresión son:

- Confianza (COF): Entendida como aquellos esfuerzos y creencias sobre que los miembros de la estructura organizacional deben de realizar por mantener relaciones interpersonales que fomenten integridad, credibilidad, seguridad, entre otras... con la finalidad de que actúen de una forma adecuada en una situación determinada.

- Participación en la toma de decisiones (PTD): Variable que se caracteriza por ser el proceso responsable de atribuir poder a aquellos elementos de la organización con una posición baja dentro de la estructura jerárquica, cuya finalidad apunta a incrementar las oportunidades de estos para que sean incluidos dentro de los procesos de toma de decisiones.

- Apoyo (AP): Elemento que se define como parte del ambiente de trabajo para los miembros de la organización, mismo que debe destacarse por la cooperación, el trabajo en equipo, con la finalidad de obtener un beneficio mutuo para los interesados y para la organización. 
- Apertura en la comunicación descendente (ACD): Referida como a la flexibilidad que existe dentro de la empresa para que el personal de jerarquía comparta información sin filtrar los mensajes mientras estos fluyen hacia abajo dentro de la estructura organizacional.

- Apertura en la comunicación ascendente (ACA): Variable que es la contraparte de la comunicación descendente, puesto que la comunicación ascendente refiere a la flexibilidad que existe en la empresa para que el personal con menor jerarquía comparta información de forma que los mensajes fluyan hacia arriba dentro de la estructura organizacional.

- Interés en las metas de alto impacto (IMA): Referido a las motivaciones que tienen los miembros de la organización por tener logros, cumplir objetivos y metas trazadas en los planes y estrategias de desarrollo y que pueden ir enfocadas en particular a elementos de desarrollo, calidad, productividad, competitividad.

Estos modelos tienen la finalidad de predecir el comportamiento del (CCO) con respecto de cada una de las variables identificadas (para ello se genera un modelo de regresión por cada variable). Toda vez que dichos modelos sean planteados, se espera que exista un aumento de la variable dependiente con respecto de las variables independientes, mismas que se espera tengan valores positivos y significativos. Lo anterior permite establecer la ecuación de regresión para cada una de las variables con respecto del (CCO) y que se representan de la siguiente manera:

$$
\mathrm{Ye}=\mathrm{B} 0+\mathrm{Bn} \mathrm{Xni}
$$

(Se sustituyen los valores [ $\mathrm{n}=$ No. Var.] para cada una de las variables [ $1-6$ ])

De lo anterior, se determina que:

- Los indicadores B evalúan la intensidad de las medias sobre los efectos de las variables explicativas (para cada caso en particular) sobre el clima de la comunicación (CCO).

- Mientras que los valores Ye representan al valor de la predicción por parte del clima de la comunicación (CCO) de las empresas del sector turístico dentro del estado de Aguascalientes estimadas por cada uno de los modelos (con respecto a cada variable independiente).

- Finalmente, el valor B0 simboliza aquellos elementos desconocidos, mientras que los valores para las respectivas X1i ... X6i son los valores para las variables independientes (COF, PTD, AP, ACD, ACA, IMA) sobre el clima de la comunicación del sector determinado.

\section{Resultados de los modelos de regresión}

Propiamente es menester indicar que todos los modelos de regresión planteados se observa la existencia de independencia en los residuales según lo evaluado por el estadístico de Durbin-Watson (ver 
tabla 1). De igual forma se encontraron valores atípicos en cada uno de los modelos, sin embargo, al no ser representativos se decidió incluirlos en el análisis para mantener la integridad del análisis. Se establece la existencia de homocedasticidad y de normalidad para todos los modelos de regresión.

Los resultados obtenidos de los modelos de regresión (ver tabla 1) indican en base a los criterios de probabilidad para la muestra de F, cuyo fundamento de entrada (para cada variable) oscila entre los rangos desde .05 hasta .10, de ello se desglosa.

Tabla 1. Comparación de los estadísticos de regresión para cada modelo

\begin{tabular}{|c|c|c|c|c|c|c|}
\hline INDICADORES & MOD No.1 & MOD No.2 & MOD No.3 & MOD No.4 & MOD No.5 & MOD No.6 \\
\hline Durbin-Watson & 2.114 & 1.775 & 1.919 & 1.863 & 2.169 & 2.053 \\
\hline $\begin{array}{l}\text { Coeficiente } \\
\text { de correlación } \\
\text { múltiple }\end{array}$ & $.712 \mathrm{a}$ & $.782 \mathrm{~b}$ & $.793 \mathrm{c}$ & $.809 \mathrm{~d}$ & $.702 \mathrm{e}$ & $.680 \mathrm{f}$ \\
\hline $\begin{array}{l}\text { Coeficiente de } \\
\text { determinación } \\
\text { R2 }\end{array}$ & .506 & .612 & .628 & .655 & .492 & .462 \\
\hline R2 Ajustado & .505 & .610 & .627 & .653 & .491 & .460 \\
\hline Error típico & .28671 & .25436 & .24888 & .23979 & .29075 & .29942 \\
\hline $\begin{array}{l}\text { No. de Observa- } \\
\text { ciones }\end{array}$ & 260 & 260 & 260 & 260 & 260 & 260 \\
\hline
\end{tabular}

${ }^{* * *}$ Nota: Valores correspondientes a los modelos de regresión en función de los predictores: (constante), con respecto de: a) Confianza (COF), b) Participación en la toma de decisiones (PTD). c) Apoyo (AP). d) Apertura en la comunicación descendente (ACD). e) Apertura en la comunicación ascendente (ACA). f) Interés en las metas de alto impacto (IMA). Variable dependiente (CCO).।

Fuente. Elaboración propia con base en trabajo de campo.

La tabla comparativa muestra los valores obtenidos para cada uno de los modelos de regresión, observando que los valores obtenidos se pueden considerar relativamente favorables para cada uno de los modelos de regresión. Observando la tabla anterior, es posible indicar que de acuerdo con lo establecido por Cohen, Cohen, West y Aiken, (2003) existe un efecto de gran tamaño para cada uno de los modelos de regresión. Propiamente se puede indicar que la variable clima de la comunicación (CCO) se encuentra determinada particularmente con cada una de las variables independientes (COF, PTD, AP, ACD, ACA, 
IMA), puesto que se muestra la existencia en una derivación efectiva de regresión altamente significativa para las variables expresadas para cada modelo en particular con respecto del (CCO).

Lo anterior se traduce en la información recopilada para el análisis de la varianza y del comparativo para cada uno de los modelos de regresión (ver tabla 2):

Tabla 2. Análisis de la Varianza para cada modelo de regresión

\begin{tabular}{|c|c|c|c|c|c|}
\hline Valores & $\begin{array}{l}\text { Suma de } \\
\text { cuadrados }\end{array}$ & gl & $\begin{array}{c}\text { Media cuadrá- } \\
\text { tica }\end{array}$ & F & Sig. \\
\hline $\begin{array}{l}\text { Modelo } 1 \\
\quad \text { Regresión } \\
\text { Residuo } \\
\text { Total }\end{array}$ & $\begin{array}{l}21.767 \\
21.209 \\
42.976\end{array}$ & $\begin{array}{c}1 \\
258 \\
259\end{array}$ & $\begin{array}{c}21.767 \\
.082\end{array}$ & 264.785 & $.000 \mathrm{a}$ \\
\hline $\begin{array}{l}\text { Modelo } 2 \\
\quad \text { Regresión } \\
\text { Residuo } \\
\text { Total }\end{array}$ & $\begin{array}{l}26.284 \\
16.692 \\
42.976\end{array}$ & $\begin{array}{c}1 \\
258 \\
259\end{array}$ & $\begin{array}{c}26.284 \\
.065\end{array}$ & 406.260 & $.000 b$ \\
\hline $\begin{array}{l}\text { Modelo } 3 \\
\text { Regresión } \\
\text { Residuo } \\
\text { Total }\end{array}$ & $\begin{array}{l}26.994 \\
15.981 \\
42.976\end{array}$ & $\begin{array}{c}1 \\
258 \\
259\end{array}$ & $\begin{array}{c}26.994 \\
.062\end{array}$ & 435.795 & $.000 c$ \\
\hline $\begin{array}{l}\text { Modelo } 4 \\
\text { Regresión } \\
\text { Residuo } \\
\text { Total }\end{array}$ & $\begin{array}{l}28.140 \\
14.835 \\
42.976\end{array}$ & $\begin{array}{c}1 \\
258 \\
259\end{array}$ & $\begin{array}{c}28.140 \\
.058\end{array}$ & 489.384 & $.000 \mathrm{~d}$ \\
\hline $\begin{array}{l}\text { Modelo } 5 \\
\text { Regresión } \\
\text { Residuo } \\
\text { Total }\end{array}$ & $\begin{array}{l}21.165 \\
21.811 \\
42.976\end{array}$ & $\begin{array}{c}1 \\
258 \\
259\end{array}$ & $\begin{array}{c}21.165 \\
.085\end{array}$ & 250.362 & $.000 \mathrm{e}$ \\
\hline $\begin{array}{l}\text { Modelo } 6 \\
\text { Regresión } \\
\text { Residuo } \\
\text { Total }\end{array}$ & $\begin{array}{l}19.845 \\
23.130 \\
42.976\end{array}$ & $\begin{array}{c}1 \\
258 \\
259\end{array}$ & $\begin{array}{c}19.845 \\
.090\end{array}$ & 221.361 & $.000 f$ \\
\hline
\end{tabular}

Esto permite identificar que todos los modelos de regresión obtenidos tienen un valor positivo correspondiente al apartado de F, esto indica que las variables independientes (COF, PTD, AP, ACD, ACA, IMA) influyen de manera positiva-significativa $(\mathrm{p}<0.005)$ en el clima de la comunicación $(\mathrm{CCO})$, lo que se traduce en que todas las variables independientes poseen el mismo comportamiento que la variable dependiente. Observando los valores encontrados en la tabla 1, propiamente se puede indicar que, si se comparan los modelos de forma aislada (considerando cada variable de forma individual) el modelo 4 $(A C D)$ es el que mayor incidencia tiene con respecto del (CCO), mientras que modelo 5 (ACA) es el que menor incidencia presenta en el (CCO). 
En base a los resultados en los modelos de regresión propuestos, se observa que si se parte del origen situado en (Bn), por cada incremento en las variables de los modelos (COF, PTD, AP, ACD, ACA, IMA), se incide en el (CCO) en esa misma proporción, siguiendo esa lógica, se representan las ecuaciones de regresión para cada modelo de la siguiente manera:

$$
\begin{aligned}
& (\text { Modelo } 1)--\mathrm{Ye}=\mathrm{B} 0+\mathrm{B} 1 \mathrm{X} 1 \mathrm{i} \text { Se sustituye por: } \mathrm{Ye}=2.157+(.487) \mathrm{COF} \\
& (1) \\
& (\text { Modelo 2) }-\mathrm{Ye}=\mathrm{B} 0+\mathrm{B} 1 \mathrm{X} 1 \mathrm{i} \text { Se sustituye por: } \mathrm{Ye}=1.876+(.551) \mathrm{PTD}
\end{aligned}
$$

En función de los valores obtenidos (ver tabla 3), con respecto a los coeficientes estandarizados (betas) se puede observar que los valores obtenidos propiamente son elevados, propiamente se desglosa el análisis de los resultados para cada uno de los factores:

- El factor 1.- Confianza (COF): se encuentra un valor para el coeficiente (beta) $B=$ .712. Esto se traduce en niveles altos de confianza dentro de la estructura organizacional, esto se traduce en que la percepción de los miembros de la organización considera que existen relaciones interpersonales integras, seguras y de calidad, además de la creencia de que estos actuaran de forma adecuada según se lo requiera.

- El factor 2.- Participación en la toma de decisiones (PTD): Se posiciona con un valor en el coeficiente (beta) $B=.782$. Esto significa que de forma general el proceso de comunicación dentro de la organización es el adecuado, puesto que existen canales de comunicación adecuados para consulta de información (indistintamente de la dirección de esta), adicionalmente el personal de todos los niveles es informado y consultado sobre aspectos organizacionales con respecto del establecimiento de objetivos y metas.

- El factor 3.- Apoyo (AP): Se posiciona con un valor en el coeficiente (beta) $ß=.793$. Esto se traduce en un ambiente de trabajo adecuado para los trabajadores, mismos que perciben un alto nivel de cooperación, trabajo en equipo, dicho ambiente de trabajo se caracteriza por poseer una atmosfera de sinceridad y franqueza sin tomar en cuenta la posición dentro de la estructura organizacional.

- El factor 4- Apertura en la comunicación descendente (ACD): Posee un valor en el coeficiente (beta) igual a $\beta=.809$. Esto significa que todo el personal recibe información que mejora sus habilidades para coordinar su trabajo con el de los demás departamentos, así como un alto nivel de interés por parte de la estructura jerárquica hacia con los de menor poder, adicionalmente se percibe que existe un alto nivel de flexibilidad en el manejo, intercambio y flujo de información (con la salvedad de aquella que comprometa "en términos de seguridad e integridad" a la organización o alguno de sus miembros) hacia los miembros que lo requieran y cuya relación sea en apego directo a su labor inmediata. 
- El factor 5.- Apertura en la comunicación ascendente (ACA): Posee un valor en el coeficiente (beta) igual a $ß=.702$. Lo anterior armoniza con elementos de confianza y de apoyo, puesto que este factor se relaciona con la percepción que tiene el personal de poder dentro de la jerarquía y con la información que recibe de sus subordinados, esto se traduce en altos niveles de colaboración y flexibilidad organizacional. Adicionalmente el personal con alto nivel de poder dentro de la jerarquía mantiene apertura hacia con los demás miembros de la organización.

- El factor 6.- Interés en las metas de alto impacto (IMA): Posee un valor en el coeficiente (beta) igual a $\beta=.680$. Este factor destaca la motivación que tiene el personal de la empresa y los esfuerzos de estos por tratar de alcanzar/lograr aquellos objetivos y metas propuestos para el desarrollo de la empresa. Se destaca particularmente que el personal se encuentra comprometido hacia dichas metas y objetivos, adicionalmente se percibe que elementos como el bienestar, la integridad del personal y sus necesidades se son elementos vitales para alcanzar un mejor desempeño organizacional, además de un nivel alto de eficacia y efectividad organizacional.

Tabla 3. Comparativo de los coeficientes de los modelos de regresión

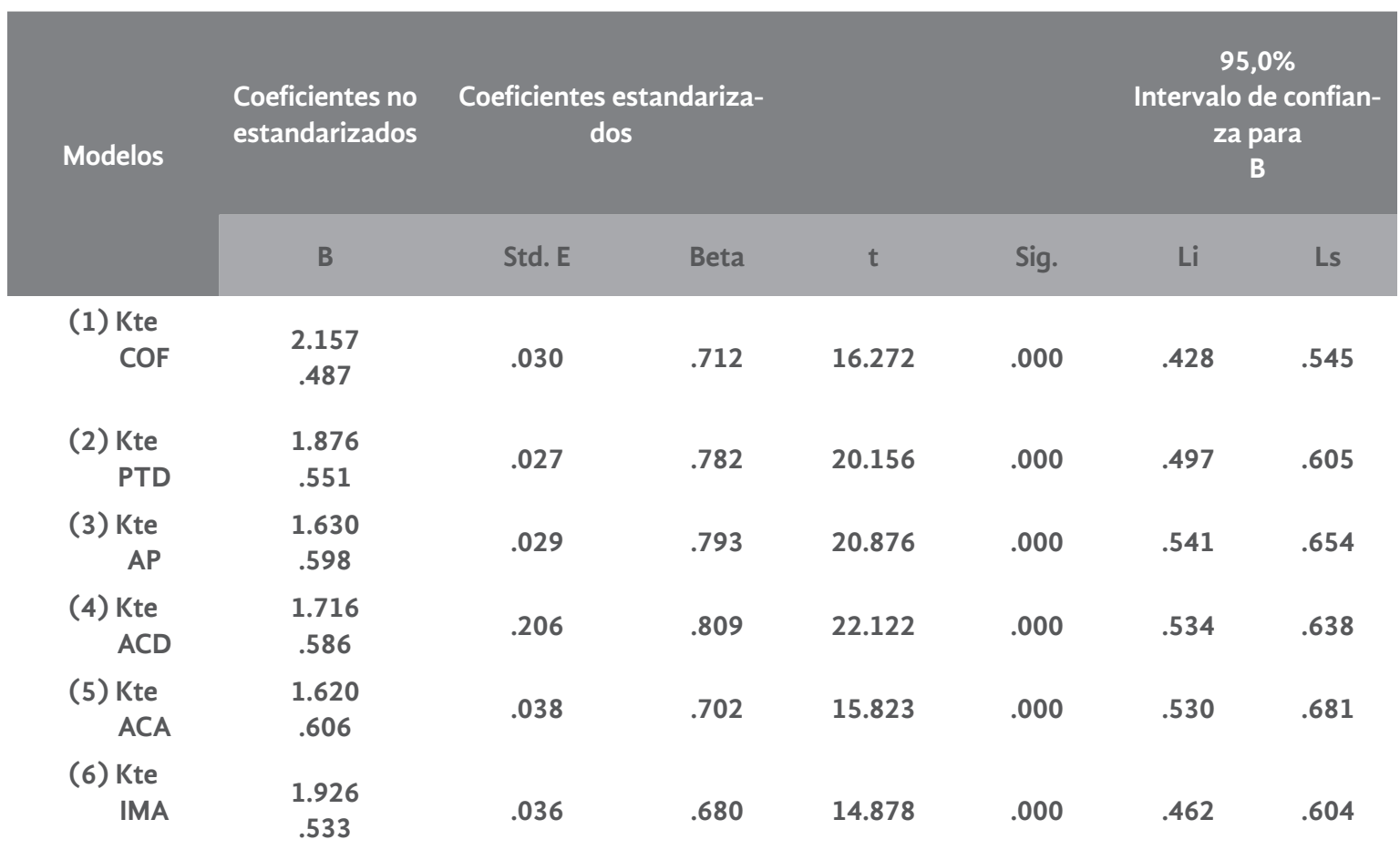

***Nota: Variable dependiente: Clima de la Comunicación (CCO). Error Estándar (Std. E). Límite Inferior (Li). Limite Superior (Ls). Valor Constante (Kte). Confianza (COF). Participación en la toma de decisiones (PTD). Apoyo (AP). Apertura en la comunicación descendente (ACD). Apertura en la comunicación ascendente (ACA). Interés en las metas de alto impacto (IMA).

Fuente. Elaboración propia con base en trabajo de campo. 
Los resultados derivados de los modelos de regresión sobre la percepción del personal directivo/gerencial de las Pymes del sector turístico dentro del estado de Aguascalientes, permiten determinar la correspondencia entre las variables: a) Confianza (COF), b) Participación en la toma de decisiones (PTD). c) Apoyo (AP). d) Apertura en la comunicación descendente (ACD). e) Apertura en la comunicación ascendente (ACA).f) Interés en las metas de alto impacto (IMA), con respecto de la variable dependiente (CCO). Con ello en mente se establece la posible contrastación de las hipótesis de investigación planteadas y con los resultados obtenidos es posible aceptar todas las hipótesis, lo que indica que:

- H1: Existe relación positiva significativa entre la confianza (COF) y el clima de la comunicación (CCO) en las empresas del sector turístico dentro del estado de Aguascalientes.

- H2: Existe relación positiva significativa entre la participación en la toma de decisiones (PTD) y el clima de la comunicación (CCO) en las empresas del sector turístico dentro del estado de Aguascalientes.

- H3: Existe relación positiva significativa entre el apoyo (AP) y el clima de la comunicación (CCO) en las empresas del sector turístico dentro del estado de Aguascalientes.

- H4: Existe relación positiva significativa entre la apertura en la comunicación descendente (ACD) y el clima de la comunicación (CCO) en las empresas del sector turístico dentro del estado de Aguascalientes.

- H5: Existe relación positiva significativa entre la apertura en la comunicación ascendente (ACA) y el clima de la comunicación (CCO) en las empresas del sector turístico dentro del estado de Aguascalientes.

- H6: Existe relación positiva significativa entre el Interés en las metas de alto impacto (IMA) y el clima de la comunicación (CCO) en las empresas del sector turístico dentro del estado de Aguascalientes.

Los resultados obtenidos mediante las pruebas de hipótesis realizadas identifican la importancia de todas las variables como elementos que ejercen influencia dentro del clima de la comunicación dentro del contexto determinado. Si bien es cierto que elementos intangibles como la comunicación o la cultura o el clima organizacionales por sí solos no contribuyen directamente en elementos tangibles dentro de la operación de las organizaciones (Ali y Patnaik, 2014; Barney, 1991; Buckley, Pass y Prescott, 1988; Hartnell, Ou y Kinicki, 2011; Sánchez-Gutiérrez, Mejía-Trejo, Vargas-Barraza y Vázquez-Ávila, 2016), sin embargo, estos elementos intangibles pueden ser facilitar la relación entre variables que si impactan directamente en el desarrollo de las empresas (Barney, 1986; Buckley et al., 1988).

Es por ello que desde hace tiempo se ha logrado establecer que, en conjunto otras variables organizacionales, algunos componentes del clima de la comunicación podrían tener un impacto positivo en elementos como el desempeño (Snyder y Morris, 1984), productividad (Clampitt y Downs, 1993), efectividad organizacional (Kerr, Ostaszkiewicz, Dunning y Martin, 2020), que incluso si se relacionan con otros factores pueden tener un impacto en la sustentabilidad y en la competitividad de las organizaciones (Cantele y Cassia, 2020). Por todo lo anterior, es importante considerar que diversas herramientas de la comunicación permiten obtener un mejor ambiente de comunicación, así como una mejora consi- 
derable en la efectividad del proceso de comunicación dentro de la organización, sin importar quienes intercambien mensajes, ni el sentido que la información tenga y mucho menos el canal que se use para transmitir los mensajes (Pérez-Rolo, 2012).

\section{Discusión y conclusiones}

El estado de Aguascalientes se encuentra ante distintos retos propios de contextos donde la oferta de servicios turísticos es más alta que la demanda de estos, lo que posiciona al estado dentro de aquellos contextos altamente competitivos, cuyo mercado es cada vez más exigente y especifico en la manera en la que satisface sus necesidades. Por ello, tanto la comunidad científica como las organizaciones y distintos organismos relacionados a las actividades turísticas en el estado han concentrado sus esfuerzos por el desarrollo de recursos que permitan comprender de una mejor forma aquellos fenómenos que acechan a las empresas de dicho sector.

Esta investigación se basa en la suposición de que los problemas a los que se enfrentan en la actualidad dichas empresas pueden ser resueltos, sino al menos permitirles ampliar su visión sobre la forma en la que dichas organizaciones compiten por mantener o aumentar su posicionamiento dentro del mercado de las Pymes del sector turístico del estado de Aguascalientes. Los resultados permiten observar que aquellas organizaciones cuyas prácticas para mejorar el ambiente de comunicación dentro de su empresa se orienten: (1) al aumento en la confianza de los miembros de la empresa, (2) así como a la colaboración y la participación de estos en el proceso de la toma de decisiones, (3) al apoyo por parte de la estructura jerárquica con respecto de aquellos miembros de menor poder, (4) a la flexibilidad del intercambio de comunicación (indistintamente de la orientación de esta) y (5) al desarrollo por el interés de los miembros en el logro de metas y objetivos, obtendrán mejores resultados en el clima de la comunicación que aquellas que no lo hagan.

El clima organizacional es uno de los elementos con mayor importancia dentro del éxito en el desarrollo e implementación de distintas prácticas, estrategias y metodologías organizacionales cuyo objetivo gire alrededor del incremento en el desempeño, la productividad, la mejora continua y la calidad tanto de los grupos como de la empresa, de la misma manera existen beneficios del manejo adecuado del clima organizacional en términos particulares, propiamente referidos al incremento en la cooperación, la participación y la proactividad de los individuos (Brunet, 1992). Es importante recalcar que el clima de la comunicación es un elemento que permite (en cierta medida) modificar las actitudes y comportamientos de los miembros de la organización, por lo que es prudente considerar que dicho ambiente debe de mantenerse dentro de los parámetros aceptados para los estándares de la organización (Chan et al., 2017; Hulbert et al., 1987).

Si se considera que el clima de la comunicación es un factor determinante en el desarrollo de la identificación organizacional, así como la disposición de los empleados por querer pertenecer a la empresa, es fundamental que este elemento sea el adecuado y se desarrolle de la mejor manera (Smidts et al., 2001). Este mismo autor indica que esto era posible puesto que por medio de esta identificación se invita al empleado a participar activamente dentro del proceso de la toma de decisiones (por el interés que tiene este por el correcto desarrollo de la empresa). Considerando lo expuesto a lo largo de este trabajo es importante recalcar que si bien la comunicación no es un elemento que propiamente impacte en al- 
gún elemento de desarrollo y desempeño organizacional, es un elemento que se encuentra fuertemente correlacionado dentro de las prácticas y en el correcto desarrollo e implementación de estrategias que permiten la mejora dentro de la organización (Dawson, Madera, Neal y Chen, 2012).

\section{Referencias}

Aburto, H. I. y Bonales, J. (2011). Habilidades directivas: Determinantes en el clima organizacional. Investigación y Ciencia, 19(51), 41-49.

Ali, A. y Patnaik, B. (2014). Influence of organizational climate and organizational culture on managerial effectiveness: An inquisitive study (Vol. 1).

Álvarez, J. (2007). Comunicación Interna, la Estrategia del Exito. Razón y Palabra, 56.

Arráez, R. B., Osuna, M. T. y Peris, G. S. (2014). La percepción sobre la función de la comunicación corporativa en los sectores tradicionales. Pensar la Publicidad. Revista Internacional de Investigaciones Publicitarias, 7(2). DOI: https://doi.org/10.5209/rev_PEPU.2013.v7.n2.46179.

Barney, J. B. (1991). Firm Resources and Sustained Competitive Advantage. Journal of Management, 17(1), 99-120. DOI: https://doi.org/10.1177/014920639101700108.

Barney, J. B. (1986). Organizational culture - can it be a source of sustained competitive advantage. Academy of Management Review, 11(3), 656-665. DOI: https://doi.org/10.2307/258317.

Bartoli, A. (1992). Comunicación y Organización. España: Paidós Ibérica.

Bowers, D. y Taylor, J. (1970). The Survey of Organizations: Toward a Machine-Scored, Standardized Questionnaire Instrument. Michigan University Ann Arbor Institute for Social Research. DOI: https:// doi.org/AD0714179.

Brownell, J. (1994). The Performance Appraisal Interview: A Multi-Purpose Communication Assignment. The Bulletin of the Association for Business Communication, 57(2), 11-21. DOI: https://doi. org/10.1177/108056999405700202.

Brunet, L. (1992). El clima de trabajo en las organizaciones: definición, diagnóstico y consecuencias. México: Trillas.

Brunet, L. (2007). El clima de trabajo en las organizaciones: definición, diagnóstico y consecuencias. México: Trillas.

Brunet, L. (2011). El Clima de Trabajo en las Organizaciones. México: Trillas.

Buckley, P. J., Pass, C. L. y Prescott, K. (1988). Measures of international competitiveness: A critical survey. Journal of Marketing Management, 4(2), 175-200. DOI: https://doi.org/10.1080/026725 7X.1988.9964068.

Cabrera, M. Á. (2015). La Toma de Decisiones en Comunicación Organizacional (Universidad Rey Juan Carlos). Recuperado de: https://eciencia.urjc.es/handle/10115/13729.

Cabrera, N. (2003). Clima organizacional, deseo y demanda. Estudio realizado en GESTA. Universidad de La Habana, Cuba.

Cantele, S. y Cassia, F. (2020). Sustainability implementation in restaurants: A comprehensive model of drivers, barriers, and competitiveness-mediated effects on firm performance. International Journal of Hospitality Management, 87, 102510. DOI: https://doi.org/https://doi.org/10.1016/j.ijhm.2020.102510. Cervera, A. (2004). Comunicación Total (3a ed.). ESIC Editorial. 
Chan, W. W., Chow, C. W., Loi, R. y Xu, A. J. (2017). Turning Hotel Employees Into Brand Champions: The Roles of Well-Connected Leaders and Organizational Identification. Journal of Hospitality \& Tourism Research, 42(8), 1232-1253. DOI: https://doi.org/10.1177/1096348017744017.

Clampitt, P. G. y Downs, C. W. (1993). Employee Perceptions of the Relationship Between Communication and Productivity: A Field Study. The Journal of Business Communication, 30(1), 5-28. DOI: https:// doi.org/10.1177/002194369303000101.

Cohen, J., Cohen, P., West, S. G. y Aiken, L. S. (2003). Applied multiple regression/correlation analysis for the behavioral sciences (3rd ed.). Lawrence Erlbaum Associates.

Cruz, Y. (2007). Estudio del clima organizacional en la Corporación COPEXTEL, SA División Territorial Pinar del Río. La Habana, Cuba.

Daradjat, A. y Sugiyanto, E. (2017). Employee participation and organizational communication in mitigating industrial relations conflict: Evidence form district of bandung, west Java province. International Journal of Applied Business and Economic Research, 15(26), 11-23.

Dawson, M., Madera, J. M., Neal, J. A. y Chen, J. (2012). The Influence of Hotel Communication Practices on Managers' Communication Satisfaction with Limited English-Speaking Employees. Journal of Hospitality \& Tourism Research, 38(4), 546-561. DOI: https://doi.org/10.1177/1096348012461552.

Dessler, G. (1993). Organización y Administración: enfoque situacional (No. 658 DESSo).

Diallo, A. y Thuillier, D. (2005). The success of international development projects, trust and communication: an African perspective. International Journal of Project Management, 23(3), 237-252. DOI: https://doi.org/https://doi.org/10.1016/j.ijproman.2004.10.002.

Duran, P. (2002). M.E.P.T: Mejores empresas para trabajar. La importancia del clima organizacional en el éxito de las empresas. Recuperado de: https://www.gestiopolis.com/caracteristicas-mejores-empresaspara-trabajar-mept/.

Escuela del Partido «Ñico López». (s/f). Maestría en Consultoría Gerencial. Curso 2. Cultura organizacional. Escuela del Partido «Ñico López».

Falcione, R. L. (1974). Communication climate and satisfaction with immediate supervision. Journal of Applied Communication Research, 2(1), 13-20. DOI: https://doi.org/10.1080/00909887409360190.

Fernández, C. (1997). La Comunicación en las organizaciones. México: Trillas.

Forehand, G. A. y Von Haller, G. (1964). Environmental variation in studies of organizational behavior. Psychological Bulletin, 62(6), 361-382. DOI: https://doi.org/10.1037/h0045960.

Gómez, D. M. y Sarsosa, K. (2011). Características de la cultura organizacional y comunicación interna en una comercializadora de lácteos de Cali. Pensamiento Psicológico, 9(17), 57-67.

Guillén del Campo, M. (2013). Clima organizacional en la Editorial Ciencias Médicas a partir del análisis de dos de sus dimensiones. Revista Cubana de Salud Pública, 39, 242-252.

Guzley, R. M. (1992). Organizational Climate and Communication Climate: Predictors of Commitment to the Organization. Management Communication Quarterly, 5(4), 379-402. DOI: https://doi.org/10.11 77/0893318992005004001.

Halpin, A. W. y Croft, D. B. (1963). The organizational climate of schools. Administrator's Notebook, 11(7), 22.

nR José Guadalupe Magdaleno Elizondo, Ismael Manuel Rodríguez Herrera y Diana Elizabeth Hernández-Ochoa 
Hartnell, C. A., Ou, A. Y. y Kinicki, A. (2011). Organizational culture and organizational effectiveness: A meta-analytic investigation of the competing values framework's theoretical suppositions. Journal of Applied Psychology, 96(4), 677-694. DOI: https://doi.org/10.1037/a0021987.

Hassan, B. y Maqsood, A. (2012). Relationship between organizational communication climate and interpersonal conflict management. Pakistan journal of psychology, 42, 23-41.

Hernández, M. A., Mendoza, J. y González, L. (2008). Construcción y validez del instrumento de cultura organizacional y competitividad (ICOC). En Estableciendo puentes en una economía global (p. 9). Escuela Superior de Gestión Comercial y Marketing, ESIC.

Hidayat, R. y Hanisha, I. (2019). The Effect Of Organizational Communication Climate On Performance Of Employees. En Bright Pln Batam BT - 1st International Conference on Applied Economics and Social Science (ICAESS 2019). DOI: https://doi.org/https://doi.org/10.2991/icaess-19.2019.60.

Hulbert, J., Gibbs, M., Hewing, P. y Underwood, R. (1987). How to Teach Interpersonal Communication Techniques in a Basic Business Communication Class. The Bulletin of the Association for Business Communication, 50(4), 24-28. DOI: https://doi.org/10.1177/108056998705000409.

Ivancevich, J. M., Konopaske, R. y Matteson, M. T. (2006). Comportamiento organizacional. España: McGraw-Hill.

Kahle, E. (2002). Implications of "New Economy" Traits for the Tourism Industry. Journal of Quality Assurance in Hospitality \& Tourism, 3(3-4), 5-23. DOI: https://doi.org/10.1300/J162v03n03_02.

Keenan, J. P. (1988). Communication Climate, Whistle-blowing, and the First-Level Manager: A Preliminary Study. Academy of Management Proceedings, 1, 247-251. DOI: https://doi.org/10.5465/ ambpp.1988.4981099.

Kerr, D., Ostaszkiewicz, J., Dunning, T. y Martin, P. (2020). The effectiveness of training interventions on nurses' communication skills: A systematic review. Nurse Education Today, 89, 104405. DOI: https:// doi.org/https://doi.org/10.1016/j.nedt.2020.104405.

Krajnakova, E., Navikaite, A. y Navickas, V. (2015). Paradigm Shift of Small and Medium-Sized Enterprises Competitive Advantage to Management of Customer Satisfaction. Inzinerine Ekonomika-Engineering Economics, 26(3), 327-332. DOI: https://doi.org/10.5755/j01.ee.26.3.6608.

Krohn, F. B. (1981). Communication climate. Medical Group Management, 28(1), 51-53.

Kwofie, T., Aigbavboa, C. y Machethe, S. (2019). Nature of communication performance in non-traditional procurements in South Africa. Engineering, Construction and Architectural Management, 26(10), 2264-2288. DOI: https://doi.org/10.1108/ECAM-02-2018-0044.

Likert, R. y Likert, J. G. (1986). Nuevas formas para solucionar conflictos. México: Trillas.

Litwin, G. H. y Stringer, R. A. (1968). Motivation and Organizational Climate of Research. Graduate School of Business Administration, Harvard University.

Mazzei, A. (2010). Promoting active communication behaviours through internal communication. Corporate Communications: An International Journal, 15(3), 221-234. DOI: https://doi. org/10.1108/13563281011068096.

Mbatha, B. (2012). Exploring the potential of electronic commerce tools in South African SME tourism service providers. Information Development, 29(1), 10-23. DOI: https://doi.org/10.1177/0266666912452270.

nR José Guadalupe Magdaleno Elizondo, Ismael Manuel Rodríguez Herrera y Diana Elizabeth Hernández-Ochoa 
Mohammed, R. y Hussein, A. (2013). Communication climate and organizational performances: a comparison studies between two public organizations. Faculty of Communication and Modern Languages University Utara Malaysia.

Montoya, J. L. (2009). El diseño y uso de indicadores de comunicación en las organizaciones antioqueñas. Anagramas Rumbos y Sentidos de la Comunicación, 7(14), 0. DOI: https://doi.org/10.22395/angr. v7n14a5.

Moreno, A., Arbeláez, S. y Calderón, L. (2014). Implementación de herramientas de comunicación interna como generadoras de cambios en las PyMEs. Razón y palabra, 18(88), 27.

Neves, P. y Eisenberger, R. (2012). Management Communication and Employee Performance: The Contribution of Perceived Organizational Support. Human Performance, 25(5), 452-464. DOI: https://doi.or g/10.1080/08959285.2012.721834.

Noknoi, C., Boripunt, W. y Ngowsiri, S. (2011). Organisational communication for becoming an autonomous university: The case of Thaksin University. The International Journal of Interdisciplinary Social Sciences: Annual Review, 6, 155-166. DOI: https://doi.org/10.18848/1833-1882/CGP/v06i02/52023.

Nordin, S. M., Sivapalan, S., Bhattacharyya, E., Ahmad, H. H. y Abdullah, A. (2014). Organizational Communication Climate and Conflict Management: Communications Management in an Oil and Gas Company. Procedia - Social and Behavioral Sciences, 109, 1046-1058. DOI: https://doi.org/https://doi. org/10.1016/j.sbspro.2013.12.587.

Nosnik, A. (1998). ¿Por qué la comunicación es relevante a la empresa? En Rebeil y Ruiz-Sandoval (Coords.), El poder de la comunicación en las organizaciones.

Nosnik, A. (2003). Comunicación productiva: un nuevo enfoque teórico. Razón y Palabra, 34(8), 12.

Nosnik, O. A. (1996). Comunicación y gestión organizacional: ciclo de conferencias. Universidad Autónoma de Bucaramanga, UNAB.

Nurlita, I. (2012). Investigation of organizational communication climate at bhayangkara surabaya university using communication climate inventory (CCI) method. Academic Research International, 3(2), 259-264. DOI: https://doi.org/ISSN-L: 2223-9553, ISSN: 2223-9944.

Ottenbacher, M. y Gnoth, J. (2005). How to Develop Successful Hospitality Innovation. Cornell Hotel and Restaurant Administration Quarterly, 46(2), 205-222. DOI: https://doi.org/10.1177/0010880404271097. Pace, W. y Faules, D. (1993). Climate of Communication. En P. Hall (Ed.), Organizational Communication ( $3^{\mathrm{a}}$ ed.). E.U.A.: Prentince Hall.

Pace, R. W., Faules, D. F. y Mulyana, D. (2010). Komunikasi organisasi: strategi meningkatkan kinerja perusahaan. PT Remaja Rosdakarya.

Park, W. W. y Kim, S. (2012). The Need of Leader-Subordinate Reciprocal Dyadic Trust to Build the Subordinate's Trust in the Organization: The Case of Korean Air Pilots. The International Journal of Aviation Psychology, 22(2), 97-119. DOI: https://doi.org/10.1080/10508414.2012.663234.

Parra, E. y Rincón, Y. (2009). Comunicación pro acuerdo: Negociar ante el conflicto. Frónesis, 16(2), 249-273. Patterson, M. G., West, M. A., Shackleton, V. J., Dawson, J. F., Lawthom, R., Maitlis, S., Robinson, D. L. y Wallace, A. M. (2005). Validating the organizational climate measure: Links to managerial practices, productivity and innovation. Journal of Organizational Behavior, 26(4), 379-408. DOI: https://doi. org/10.1002/job.312.

nR José Guadalupe Magdaleno Elizondo, Ismael Manuel Rodríguez Herrera y Diana Elizabeth Hernández-Ochoa 
Patterson, P. (2016). Retrospective: tracking the impact of communications effectiveness on client satisfaction, trust and loyalty in professional services. Journal of Services Marketing, 30(5), 485-489. DOI: https://doi.org/10.1108/JSM-05-2016-0190.

Peña-Acuña, B., Caldevilla-Domínguez, D. y Batalla, P. (2017). Tres casos de empresas internacionales con éxito: estudio de las estrategias de comunicación interna. Chasqui. Revista Latinoamericana de Comunicación, 134, 0. DOI: https://doi.org/10.16921/chasqui.v0i134.2697.

Pérez-Rolo, M. (2012). Fundamentos teóricos metodológicos para realizar una campaña de relaciones públicas. Observatorio de la Economía Latinoamericana, 164, 13. DOI: https://doi.org/ISSN 1696-8352. Pérez, J. (2000). La aplicación de modelos de comunicación en las organizaciones. En Plaza y Valdés (Eds.), El poder de la comunicación en las organizaciones, 93-124.

Petersen, D. (2005). Setting goals measuring performance: Frequency versus severity. Professional Safety, 50(12), 43.

Peterson, D. y Pace, W. (1976). Communication climate and Organizational satisfaction. En Dalam, W. R. y Faules, D. F. (Eds.) Organizational Communication (3a ed.). E.U.A. Prentice Hall.

Phillips, N. y Brown, J. L. (1993). Analyzing communication in and around organizations: A critical hermeneutic approach. Academy of Management Journal, 36(6), 1547-1576. DOI: https://doi. org/10.2307/256821.

Pincus, J. D., Knipp, J. E. y Rayfield, R. E. (1990). Internal Communication and Job Satisfaction Revisited: The Impact of Organizational Trust and Influence on Commercial Bank Supervisors. Public Relations Research Annual, 2(1-4), 173-191. DOI: https://doi.org/10.1207/s1532754xjprr0201-4_7.

Pritchard, R. D. y Karasick, B. W. (1973). The effects of organizational climate on managerial job performance and job satisfaction. Organizational Behavior \& Human Performance, 9(1), 126-146. DOI: https://doi.org/10.1016/0030-5073(73)90042-1.

Ramadanty, S. y Martinus, H. (2016). Organizational Communication: Communication and Motivation in the Workplace. Humaniora Binus, 7(1), 77-86.

Rebeil, M. (2006). Comunicación estratégica en las organizaciones. México: Trillas.

Robbins, S. P. y Judge, T. (2013). Comportamiento Organizacional. México: Pearson Education.

Robbins, S. P. (1999). Comportamiento organizacional. México: Prentice Hall.

Robbins, S. P. y Coulter, M. (2002). Administración (7a ed.). México: Prentice Hall.

Rodríguez, G., Flores, G. y García, E. (2006). Metodología de la investigación cualitativa. México: Editorial Félix Varela.

Rodríguez, J. (2003). El factor humano en la empresa. España: Ediciones Deusto.

Rosenfeld, L. B. (1983). Communication climate and coping mechanisms in the college classroom. Communication Education, 32(2), 167-174. DOI: https://doi.org/10.1080/03634528309378526.

Sager, K. L. (2015). Looking down from above: measuring downward maintenance communication and exploring Theory X/Y assumptions as determinants of its expresión. Revista de Psicología del Trabajo y de las Organizaciones, 31, 41-50.

Salazar, J. G., Guerrero, J. C., Machado, Y. B. y Cañedo, R. (2009). Clima y cultura organizacional: dos componentes esenciales en la productividad laboral. ACIMED, 20, 67-75.

nR José Guadalupe Magdaleno Elizondo, Ismael Manuel Rodríguez Herrera y Diana Elizabeth Hernández-Ochoa 
Sánchez-Gutiérrez, J., Mejía-Trejo, J., Vargas-Barraza, J. A. y Vázquez-Ávila, G. (2016). Intellectual capital, impact factor on competitiveness: manufacturing industry SMEs in Mexico. Measuring Business Excellence, 20(1), 1-11. DOI: https://doi.org/10.1108/mbe-12-2015-0059.

Sanchis, P. I. y Bonavia, T. (2017). Análisis del Sistema de Comunicación Interna de una Pequeña Empresa. WPOM: Working Papers on Operations Management, 8(1), 9-21. DOI: https://doi.org/doi:10.4995/ wpom.v8i1.7390.

Sandoval-Caraveo, M. C. (2004). Concepto y dimensiones del clima organizacional. Hitos de Ciencias Económico Administrativas, 27, 78-82.

Segovia, M. (1999). El correo electrónico y la comunicación en las organizaciones formales (Tesis Doctoral). Universidad Complutense de Madrid, Servicio de Publicaciones.

Silva, M. (1996). El clima en las organizaciones: teoría, método e intervención. Promociones y Publicaciones Universitarias.

Smidts, A., Pruyn, A. T. y Van Riel, C. B. (2001). The impact of employee communication and perceived external prestige on organizational identification. Academy of Management Journal, 44(5), 1051-1062. DOI: https://doi.org/10.2307/3069448.

Snyder, R. A. y Morris, J. H. (1984). Organizational communication and performance. Journal of Applied Psychology, 69(3), 461-465. DOI: https://doi.org/10.1037/0021-9010.69.3.461.

Soria, R. (2008). Comunicación organizacional: un modelo aplicable a la microempresa. Teorías, Enfoques y Aplicaciones en las Ciencias Sociales, 1(1), 18.

Stewart, L. P., Gudykunst, W. B., Ting-Toomey, S. y Nishida, T. (1986). The effects of decision-making style on openness and satisfaction within Japanese organizations. Communication Monographs, 53(3), 236-251. DOI: https://doi.org/10.1080/03637758609376139.

Trombetta, J. J. y Rogers, D. P. (1988). Communication climate, job satisfaction, and organizational commitment: The Effects of Information Adequacy, Communication Openness, and Decision Participation. Management Communication Quarterly, 1(4), 494-514. DOI: https://doi.org/10.1177/0893318988001004003.

Uysal, H. T. (2019). The Effect of Managers' Trust in Subordinate on Entrepreneurial Intention: A Research on SMEs. Gaziantep University Journal of Social Sciences, 18(2), 0. DOI: https://doi.org/10.21547/ jss.436008.

Wei, Y. y Morgan, N. A. (2004). Supportiveness of Organizational Climate, Market Orientation, and New Product Performance in Chinese Firms. Journal of Product Innovation Management, 21(6), 375388. DOI: https://doi.org/10.1111/j.0737-6782.2004.00092.x.

Weihrich, H. y Koontz, H. (1993). Menedžment.

Wheeless, V. E., Wheeless, L. R. y Howard, R. D. (1983). An analysis of the contribution of participative decision making and communication with supervisor as predictors of job satisfaction. Research in Higher Education, 18(2), 145-160. DOI: https://doi.org/10.1007/BF00992059.

Zach, F. (2013). Collaboration for Innovation in Tourism Organizations: Leadership Support, Innovation Formality, and Communication. Journal of Hospitality \& Tourism Research, 40(3), 271-290. DOI: https://doi.org/10.1177/1096348013495694.

Zeffane, R., Tipu, S. A. y Ryan, J. C. (2011). Communication, commitment \& trust: Exploring the triad. International Journal of Business and Management, 6(6), 77-87. DOI: https://doi.org/10.5539/ijbm.v6n6p77.

nR José Guadalupe Magdaleno Elizondo, Ismael Manuel Rodríguez Herrera y Diana Elizabeth Hernández-Ochoa 